\title{
Report on a pilot project to introduce a publications officer
}

\author{
Kelly D. Cobey PhD MRes, James Galipeau PhD MA, Larissa Shamseer MSc, David Moher PhD MSc
}

$\longrightarrow$ oncerns about deficiencies in the reporting quality of biomedical research have been expressed for more than three decades. ${ }^{1}$ In spite of this, articles continue to pass through editorial and peer review processes and are published with critical aspects of their methods and results missing or inadequately described. Reporting biases also remain problematic. ${ }^{2-7}$ Together, these practices limit the integrity of biomedical literature and hinder reproducibility efforts. In an attempt to alleviate these problems, Moher and Altman recently proposed four potential contributory actions for journals and educational institutions to consider. ${ }^{8}$ Here, we present a description of our efforts to implement their first proposed action: the introduction of a publications officer.

The primary objective of a publications officer is to provide institutional guidance and support to researchers and trainees on how to prepare manuscripts for journal submission. ${ }^{8}$ At present, formal training on how to write biomedical manuscripts is largely absent from universities and other research institutions. Where such training does exist, it tends to be informal and may not be evidence-based. Moreover, existing training courses on academic integrity typically omit discussion of publication integrity and ethics or how to report research adequately, both of which are essential responsibilities for authors. This situation is inadequate, and lack of training on these topics may be partially to blame for the reporting deficiencies outlined above. The introduction of publications officers may represent a meaningful institutional investment to fill an important gap in support services at the back end of research.

We began a pilot project in which we hired a publications officer (K.D.C.) at our institution, The Ottawa Hospital Research Institute, and the neighbouring Children's Hospital of Eastern Ontario Research Institute. Both institutions recognized that there are problems with biomedical reporting and have sought to be innovators in developing solutions in this area. Against this background, it is unsurprising that our experience with this pilot project has been mostly positive. Discussions with relevant leadership at both institutions suggest that research- ers have similar learning needs with regard to publication processes. We have perceived a genuine appetite for publications outreach, with a particular interest in topics that include authorship policies, predatory journals, peer review and guidance on selecting a journal for manuscript submission.

Discipline research leaders across both institutions appear to support the relevance of this type of service. Interestingly, on occasion, there has been some lack of clarity regarding the scope of the publications officer position. Our impression is that several senior scientists felt that the services offered by the publications officer would be beneficial for graduate students and researchers who are at the beginning of their career development; however, senior scientists were more resistant to the perceived impact it could have on them. We hope to reach a broad spectrum of researchers through targeted seminars (e.g., speaking at retreats for senior scientists). Furthermore, within our two organizations, we found that the lag period (six months) between hiring the publications officer and starting outreach services enabled our understanding of institutional structures, establishment of relationships, building interest and scheduling outreach programs.

We are currently evaluating the publications officer position. Briefly, we intend to conduct before-and-after comparisons of self-reported knowledge and perceptions of publication practices among researchers at institutes that do and do not receive outreach from the publications officer. Planned outreach involves a series of targeted seminars (e.g., How to peer review), researcherinitiated one-on-one consultations to discuss publication questions and the development of a presub-

\section{KEY POINTS}

- Deficiencies in the reporting quality of biomedical research limit the usability of findings.

- Introducing institutional publications officers, who provide training and outreach to authors on how to write clearly and completely, as well as advice on publication topics (e.g., open access, metrics, ethics and integrity), could reduce these deficiencies.

- We describe our pilot of a publications officer and our planned monitoring framework for the position.
Competing interests: Kelly Cobey is the publications officer at the Ottawa Hospital Research Institute. No other competing interests were declared.

This article has been peer reviewed.

Correspondence to: Kelly Cobey, kcobey@toh.on.ca

CMAJ 2016. DOI:10.1503 /cmaj.151340 
mission peer review network for manuscripts. In addition, we will maintain a web page of journalology resources (www.ohri.ca/journalology) to assist researchers in keeping up to date on internal and external changes in the publication landscape. Specific methods and findings of this evaluation will be described in a future publication.

We will continue to monitor this new role by tracking and reporting changes over time so that the position evolves to serve the research community most usefully. Introducing publications officers, if proven to be worthwhile and impactful, may be one way that research institutions can contribute to adding value to the biomedical literature. Unquestionably, new models and options to incentivize complete and transparent reporting will need to be explored in tandem with this role.

Affiliations: Centre for Journalology, Clinical Epidemiology Program (Cobey, Galipeau, Shamseer, Moher), Ottawa Hospital Research Institute; School of Epidemiology, Public Health and Preventive Medicine (Cobey, Shamseer, Moher), Faculty of Medicine, University of Ottawa, Ottawa, Ont.; Department of Psychology (Cobey), University of Stirling, Stirling, UK
Contributors: Kelly Cobey drafted the manuscript. James Galipeau, Larissa Shamseer and David Moher contributed important intellectual feedback and commentary on several versions of the manuscript. All of the authors gave final approval of the version to be published and agreed to act as guarantors of the work.

\section{References}

1. Hemminki E. Study of information submitted by drug companies to licensing authorities. BMJ 1980;280:833-6.

2. Glasziou P, Meats E, Heneghan C, et al. What is missing from descriptions of treatment in trials and reviews? BMJ 2008;336: 1472-4.

3. Dwan K, Gamble C, Williamson PR, et al. Systematic review of the empirical evidence of study publication bias and outcome reporting bias — an updated review. PLoS One 2013;8: e66844.

4. Mallett S, Timmer A, Sauerbrei W, et al. Reporting of prognostic studies of tumour markers: a review of published articles in relation to REMARK guidelines. Br J Cancer 2010; 102:173-80.

5. Kilkenny C, Parsons N, Kadyszewski E, et al. Survey of the quality of experimental design, statistical analysis and reporting of research using animals. PLoS One 2009;4:e7824.

6. Chan A, Hrobjartsson A, Haahr MT, et al. Empirical evidence for selective reporting of outcomes in randomized trials. JAMA 2004;291:2457-65.

7. Saini P, Loke YK, Gamble C, et al. Selective reporting bias of harm outcomes within studies: findings from a cohort of systematic reviews. BMJ 2014;349:g6501.

8. Moher D, Altman DG. Four proposals to help improve the medical research literature. PLoS Med 2015;12:e1001864. 